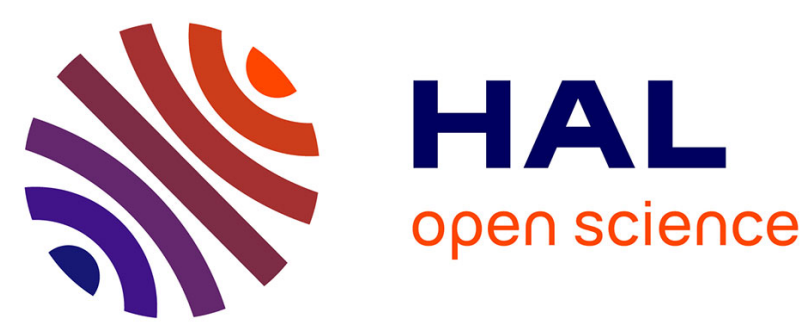

\title{
Le garrot chirurgical en pratique
}

Alain Cartigny

\section{To cite this version:}

Alain Cartigny. Le garrot chirurgical en pratique. Inter bloc, 2018, 37, pp.182 - 184. 10.1016/j.bloc.2018.06.012 . hal-03487621

\section{HAL Id: hal-03487621 \\ https://hal.science/hal-03487621}

Submitted on 20 Dec 2021

HAL is a multi-disciplinary open access archive for the deposit and dissemination of scientific research documents, whether they are published or not. The documents may come from teaching and research institutions in France or abroad, or from public or private research centers.
L'archive ouverte pluridisciplinaire HAL, est destinée au dépôt et à la diffusion de documents scientifiques de niveau recherche, publiés ou non, émanant des établissements d'enseignement et de recherche français ou étrangers, des laboratoires publics ou privés.

\section{다)(1) $(5$}

Distributed under a Creative Commons Attribution - NonCommercial| 4.0 International 
Version of Record: https://www.sciencedirect.com/science/article/pii/S024239601830095X

Manuscript_4fa88ca3796e47ae164ae72cf4f67613

\title{
Dochead technique chirurgicale
}

\section{Surtitre mise au point}

\section{Le garrot chirurgical en pratique}

\begin{abstract}
Alain Cartigny
Cadre supérieur de santé, responsable pédagogique et formateur à l'école d'infirmiers du bloc opératoire
\end{abstract}

Centre hospitalier régional universitaire de Montpellier, 1146 avenue du Père Soulas, 34295 Montpellier cedex 5, France

Adresse e-mail : a-cartigny@chu-montpellier.fr(A.Cartigny)

\section{Résumé}

La chirurgie orthopédique et traumatologique utilise encore fréquemment le garrot chirurgical. Ce dernier a beaucoup évolué, rendant son utilisation presque banale, à tel point qu'il est parfois difficile de savoir qui peut en avoir l'usage et comment s'en servir. Ce dispositif médical est-il si anodin ? Sa pose nécessite pourtant de réunir plusieurs conditions pour l'utiliser en minimisant au maximum les risques.

(C) 2018

Mots clés : dispositif médical ; garrot chirurgical ; hyperpression ; pratique réglementée ; séquelle neurologique

\section{Summary à venir}

(c) 2018

Keywords à venir

\section{T1. Notions générales}

Le garrot chirurgical est généralement un dispositif médical électrique permettant d'établir autour d'un membre, une pression circonférentielle dans le but d'interrompre la circulation sanguine, en aval du garrot et de rendre ainsi le champ opératoire exsangue. Il est constitué d'une console de contrôle de pression et de temps associée à des brassards adaptés aux membres. 


\section{T2. Réglementation}

TEG1 La pose d'un garrot chirurgical nécessite une prescription médicale, ou un protocole validé et actualisé, pour être utilisé par un infirmier de bloc opératoire ou un infirmier. Il s'agit d'un acte dépendant du rôle prescrit de l'infirmier qui ne peut être délégué à une autre catégorie de professionnel de santé.

TEG1 Le Code de la Santé publique, à l'article R4311-9, précise "L'infirmier est habilité à accomplir sur prescription médicale écrite, qualitative et quantitative, datée et signée, les actes et soins suivants, à condition qu'un médecin puisse intervenir à tout moment : [...] Application d'un garrot pneumatique d'usage chirurgical [...] » [1]. Il est également stipulé à l'article R4311-11, que "L'infirmier ou l'infirmière titulaire du diplôme d'État de bloc opératoire ou en cours de formation préparant à ce diplôme, exerce en priorité les activités suivantes : [...] Gestion des risques liées à l'activité et à l'environnement opératoire [...] » [2].

TEG1 Les indications de pose d'un garrot chirurgical sont d'obtenir un champ opératoire exsangue, de diminuer les saignements opératoires, de faciliter le geste chirurgical et de diminuer la durée de l'acte chirurgical.

\section{T2. Matériel}

TEG1 Avant son utilisation, il est nécessaire de bien connaître le fonctionnement de ce dispositif médical. Il convient donc d'avoir au préalable étudié la notice technique, de s'être assuré du bon fonctionnement du garrot, en vérifiant notamment la bonne maintenance des appareils utilisés, permettant l'étalonnage.

Il faut disposer d'un contrôle de la pression et du temps de pose, vérifier l'intégrité et la fonctionnalité du dispositif, la présence des accessoires nécessaires, et disposer de brassards de taille adaptée (rapport largeur du garrot/circonférence du membre garrotté supérieur ou égal à 0,4).

TEG1 Pour éviter tout risque d'infection, il est possible d'opter pour des brassards lavables, voire stérilisables. L'Ibode doit vérifier la propreté du dispositif et de ses accessoires.

\section{T1. Utilisation du garrot chirurgical}

TEG1 Le brassard s'appose sur une zone cutanée saine et sèche, à distance des paquets nerveux et des reliefs osseux, en gardant un espace entre le garrot et le membre. Il faut s'assurer de la protection du membre garrotté (plis, lésions cutanées), éviter l'infiltration d'antiseptique pouvant provoquer des lésions à type de brûlure (antiseptique alcoolique).

Du fait des répercussions physiologiques, il est recommandé de ne garrotter qu'un seul membre à la fois.

TEG1 Pour obtenir l'exsanguination du membre garrotté, il faut proscrire la bande d'Esmarch (en caoutchouc) qui peut générer une pression d'environ $1200 \mathrm{mmHg}$ et provoquer des 
torsions cutanées, des fractures de plaque d'athérome, libérer des ostéophytes, faire migrer des micro-emboles et entraîner une dissémination bactérienne.

TEG1 L'exsanguination peut être suffisamment efficace en pratiquant la surélévation du membre : membre supérieur à $90^{\circ}$ pendant 3 à 4 minutes, membre inférieur à $45^{\circ}$ pendant 5 à 7 minutes. Elle peut être associée à une vidange manuelle, de l'extrémité du membre vers sa racine à l'aide d’une bande Velpeau ${ }^{\circledast}$.

\section{T2. Pression}

C'est la prescription médicale qui détermine la pression de garrotage : celle-ci est variable en fonction de la taille du membre et de la largeur du garrot.

TEG1 La pression d'occlusion maximale recommandée correspond à la pression artérielle systolique (PAS) x 1,5 mmHg maximum pour un membre supérieur, et à PAS x 2,5 mmHg maximum pour un membre inférieur. L'absence de pouls distal après gonflage indique, à l'instant de contrôle, que la pression est suffisante pour bloquer la circulation artérielle. Une pression insuffisante génère le risque de garrot veineux : la circulation artérielle n'est pas arrêtée alors que la circulation veineuse l'est, ce qui provoque un gonflement et une pression croissante dans le système veineux du membre garroté ainsi qu'un saignement abondant et en nappe lors de l'incision d'un vaisseau.

TEG1 Il est recommandé alors de recommencer la procédure, en commençant par dégonfler le garrot, contrôler la tension artérielle du patient et choisir une pression de gonflage adéquate (prescription).

Car_illus1

\section{T2. Durée}

Du fait des risques, la durée de garrotage d'un membre, en continue, ne devrait pas dépasser 150 minutes, de préférence pas plus de 90 minutes pour un membre supérieur, et pas plus de 120 minutes, pour un membre inférieur. Il est possible d'utiliser, pour les chirurgies les plus longues, une technique de séquençage, alternant une période de garrotage ne dépassant les durées ci-dessus, puis une période de reperfusion du membre garrotté d'au moins 15 minutes, puis une nouvelle période de garrotage du membre. Il peut ainsi être prévu une période de reperfusion de 15 minutes après 90 minutes de garrot et une reperfusion de 20 minutes après deux heures de garrotage.

Dans tous les cas, il est important d'organiser la surveillance du garrotage en vérifiant régulièrement la pression du manomètre (annonce des temps de garrot) et le saignement local.

\section{T2. Dégonflage et levée}

TEG1 À la fin de la chirurgie, le dégonflage du garrot est demandé par le chirurgien, qui doit obtenir le feu vert de l'équipe d'anesthésie, quand les paramètres hémodynamiques sont stables et qu'il a été procédé à une augmentation de la ventilation en $\mathrm{O}_{2}$ pendant 5 à 10 minutes. La levée de garrot s'opère après la suture cutanée et le pansement ; parfois le chirurgien désire contrôler l'hémostase 
et la demande avant la fermeture. Il est possible de surélever le membre (pour éviter une hypovolémie relative). La levée est toujours réalisée avant la pose d'un plâtre ou d'un bandage, du fait de l'augmentation de volume du membre garrotté de $10 \%$ après dégonflage). Dans tous les cas, il est nécessaire de dégonfler le garrot rapidement, de contrôler le retour de sensation des pouls distaux.

TEG1 La traçabilité comprendra l'horaire de gonflage/dégonflage du garrot, la durée du garrotage, la pression exercée, le cas échéant la ou les périodes de reperfusion du membre, les lésions apparentes, le contrôle de la présence du pouls, la recoloration du membre et l'identification de l'opérateur.

\section{T1. Conséquences}

Les conséquences de la mise en place d'un garrot chirurgical au niveau d'un membre, qui interrompt la circulation artério-veineuse dans la région située en aval, sont celles de l'hyperpression localisée, métabolique, cutanée, musculaire, vasculaire et nerveuse.

\section{T2. Métabolique}

L'hyperpression et l'arrêt de la circulation sanguine provoquent pour les tissus du membre garroté, une hypoxie, une hypercapnie et une acidose. Les tissus ischémiés présentent un tableau de rhabdomyolyse dès la $40^{\mathrm{e}}$ minute d'ischémie.

\section{T2. Cutanée}

L'hyperpression localisée peut engendrer sur la peau, brûlures, phlyctènes, ulcérations secondaires et cisaillement (mouvement de rotation du garrot), surtout chez les sujets à la peau fragile, comme les personnes âgées et les enfants.

\section{T2. Musculaire}

La compression musculaire localisée, accompagnée d'une ischémie, d'une hypoxie, peut générer cisaillement, douleur, ischémie, perte de force et rhabdomyolyse. La biopsie du muscle Vastus lateralis lorsqu'un garrot chirurgical gonflé à 400 mmHg est appliqué, montre après 15 minutes, un œdème interstitiel accompagné d'une occlusion capillaire ; après 30 minutes, un œdème intracellulaire et un épaississement de la paroi capillaire ; après 60 minutes, la biopsie met en évidence de vastes lysosomes; après 90 minutes, toutes les fibres contiennent des lysosomes, des vacuoles et des organelles autophagiques, témoins d'une dégénération menant à la mort cellulaire [3]. 


\section{T2. Vasculaire}

TEG1 L'utilisation du garrot chirurgical et la compression vasculaire génèrent un risque de rupture, de cisaillement des vaisseaux sous-jacents ou de fracture de plaque d'athérome, de thrombose veineuse dans le lit d'aval accompagné de la survenue de micro-embols.

Les risques cardiovasculaires à la levée du garrot, alors que le volume sanguin mobilisé lors du garrotage est de $400 \mathrm{~mL}$ à $700 \mathrm{~mL}$ de sang pour un membre inférieur et $50 \mathrm{~mL}$ pour un membre supérieur, sont une augmentation de la fréquence cardiaque puis une bradycardie, une baisse significative de la tension artérielle (reperfusion du membre garroté et hypovolémie relative) puis un retour à la normale.

TEG1 L'embolie pulmonaire constitue la complication majeure à redouter lors de l'utilisation du garrot. L'utilisation de l'échographie trans-œsophagienne a mis en évidence un processus emboligène sans retentissement clinique chez 23 à $100 \%$ des patients [4].

\section{T2. Nerveuse}

La compression nerveuse et l'hyperpression localisée provoquent douleur, paresthésie, paralysie réversible ou non (dans $0,15 \%$ des cas). Après 60 minutes de garrot $(350 \mathrm{mmHg}$ ), la vitesse de conduction nerveuse se trouve diminuée.

Après une chirurgie de libération du canal carpien, à trois semaines de la chirurgie, il existe encore des anomalies à l'électromyogramme, témoignant d'un dysfonctionnement de la conduction nerveuse, avec garrot chirurgical de l'ordre de $77 \%$, et sans garrot chirurgical de l'ordre de $3 \%$ [5].

\section{T2. Contre-indications absolues}

Du fait de ces multiples conséquences, l'emploi du garrot chirurgical est strictement contre-indiqué pour les patients :

- porteurs d'un pontage vasculaire périphérique ;

- insuffisants rénaux dialysés ;

- atteints d'athérosclérose, d'artériopathies ;

- présentant des lésions infectieuses, ayant une fragilité cutanée, une fragilité excessive de la peau ;

- présentant un œdème cutané.

\section{T1. Conclusion}

L'utilisation du garrot pneumatique doit être justifiée par l'acte opératoire. C'est une pratique règlementée, un acte médical délégué faisant partie du rôle prescrit de l'infirmier. En effet, le garrot chirurgical majore durablement l'impotence fonctionnelle postopératoire, le volume du membre ischémié et la douleur postopératoire. 
Parmi les points à retenir, notons que la pression nécessaire pour occlure le lit artériel est d'autant plus faible que la largeur du garrot est importante. Le rapport entre la largeur du garrot et le diamètre du membre doit être supérieur à 0,4 . La formation de thrombus dans le lit d'aval est inhérente à la technique. Son ampleur est inversement proportionnelle à la déplétion sanguine du membre concerné.

Les poussées hypertensives au cours de l'utilisation du garrot sont liées à l'apparition retardée de phénomènes nociceptifs. Les anesthésies locorégionales bloquent plus efficacement ces variations hémodynamiques que l'anesthésie générale.

Les séquelles neurologiques au décours de l'utilisation du garrot sont rares et régressent habituellement dans les six mois qui suivent l'intervention. Elles peuvent survenir lors d'une utilisation inférieure à deux heures.

La maintenance et l'étalonnage régulier de l'appareil doit faire partie des règles de fonctionnement des blocs opératoires utilisant un garrot pneumatique.

L'anesthésiste doit être prévenu et immédiatement disponible au moment de la levée du garrot chirurgical.

\section{Références}

[1] Code de la santé publique. Article R4311-9. www.legifrance.gouv.fr/affichCodeArticle.do?cidTexte=LEGITEXT000006072665\&idArticl e=LEGIARTI000006913897

[2] Code de la santé publique. Article R4311-11. www.legifrance.gouv.fr/affichCodeArticle.do?cidTexte=LEGITEXT000006072665\&idArticl e=LEGIARTI000006913899\&dateTexte=\&categorieLien=cid

[3] Appell HJ, Glöser S, Duarte JA, Zellner A, Soares JM. Skeletal muscle damage during tourniquet-induced ischaemia. The initial step towards atrophy after orthopaedic surgery? Eur J Appl Physiol Occup Physiol. 1993;67(4):342-7.

[4] Hirota K, Hashimoto H, Kabara S, Tsubo T, Sato $Y$ et al. The relationship between pneumatic tourniquet time and the amount of pulmonary emboli in patients undergoing knee arthroscopic surgeries. Anesth Analg. 2001 Sep;93(3):776-80.

[5] Kokki H, Väätäinen U, Miettinen H, Parviainen A, Könönen M, Partanen J. Tourniquetinduced enmg changes in arthroscopic anterior cruciate ligament reconstruction. A comparison of low and high-pressure tourniquet systems. Ann Chir Gynaecol. 2000;89(4):313-7.

Déclaration de liens d'intérêts

L'auteur déclare ne pas avoir de liens d'intérêts.

\section{Illustration}

$$
\text { Car_illus1 }
$$




\section{(c) psdesign1/stock.adobe.com}

\section{Légende :}

Si la circulation artérielle n'est pas arrêtée alors que la veineuse l'est, un gonflement et une pression croissante dans le système veineux du membre garroté apparaissent ainsi qu'un saignement abondant en nappe lors de l'incision d'un vaisseau.

Près de son appel, sur 2 colonnes en haut 


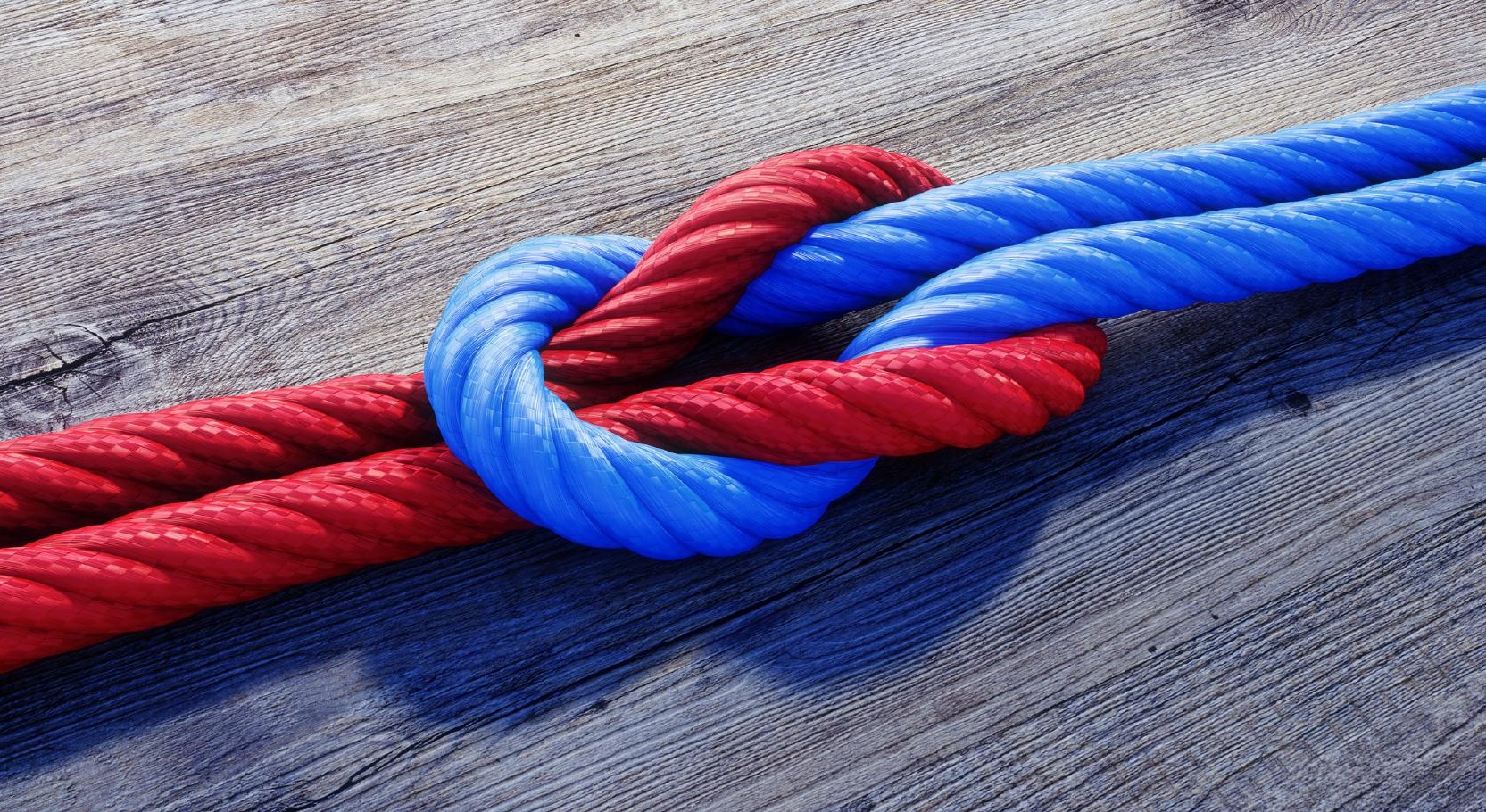

